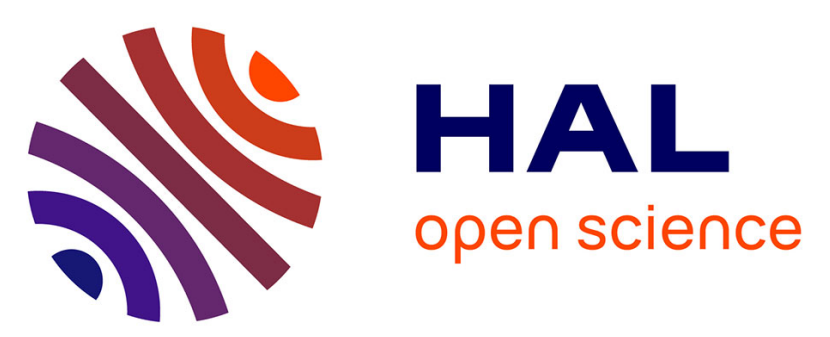

\title{
High accuracy velocity control method for the french moving-coil watt balance
}

Suat Topsu, Luc Chassagne, Darine Haddad, Yasser Alayli, Patrick Juncar

\section{To cite this version:}

Suat Topsu, Luc Chassagne, Darine Haddad, Yasser Alayli, Patrick Juncar. High accuracy velocity control method for the french moving-coil watt balance. Review of Scientific Instruments, 2004, 75 (11), pp.4824-4827. 10.1063/1.1809302 . hal-00870592

\section{HAL Id: hal-00870592 https://hal.science/hal-00870592}

Submitted on 7 Oct 2013

HAL is a multi-disciplinary open access archive for the deposit and dissemination of scientific research documents, whether they are published or not. The documents may come from teaching and research institutions in France or abroad, or from public or private research centers.
L'archive ouverte pluridisciplinaire HAL, est destinée au dépôt et à la diffusion de documents scientifiques de niveau recherche, publiés ou non, émanant des établissements d'enseignement et de recherche français ou étrangers, des laboratoires publics ou privés. 


\title{
High accuracy velocity control method for the french moving-coil watt balance
}

\author{
Suat Topcu, Luc Chassagne, Darine Haddad, and Yasser Alayli \\ LIRIS, Université de Versailles, 45 Avenue des États-Unis, 78035 Versailles, France \\ Patrick Juncar \\ BNM-INM/CNAM, 292 Rue Saint Martin, 75141 Paris, France
}

(Received 9 February 2004; accepted 20 August 2004; published 1 November 2004)

\begin{abstract}
We describe a novel method of velocity control dedicated to the French moving-coil watt balance. In this project, a coil has to move in a magnetic field at a velocity of $2 \mathrm{~mm} \mathrm{~s}^{-1}$ with a relative uncertainty of $10^{-9}$ over $60 \mathrm{~mm}$. Our method is based on the use of both a heterodyne Michelson's interferometer, a two-level translation stage, and a homemade high frequency phase-shifting electronic circuit. To quantify the stability of the velocity, the output of the interferometer is sent into a frequency counter and the Doppler frequency shift is recorded. The Allan standard deviation has been used to calculate the stability and a $\sigma_{y}(\tau)$ of about $2.2 \times 10^{-9}$ over $400 \mathrm{~s}$ has been obtained. (C) 2004 American Institute of Physics. [DOI: 10.1063/1.1809302]
\end{abstract}

\section{INTRODUCTION}

At present, the base units of the SI are related to atomic properties or fundamental constants, except for the unit of mass. Actually, the kilogram is still defined in relation to an artifact, a platinium-iridium cylinder kept at the Bureau International des Poids et Mesures (BIPM). The results of periodic comparisons between national kilogram prototypes and the standard unit of mass have called the definition of this unit into question as a mean drift of $5 \times 10^{-8} \mathrm{~kg}$ has been observed over the last century. ${ }^{1}$ One strategy to improve the status of the kilogram is the watt balance project which relates the unit of mass to Planck's constant via electrical energy. ${ }^{2-5}$ One can show ${ }^{6}$ that if the electrical quantities are measured using the Josephson effect ${ }^{7}$ and the quantum Hall effect, ${ }^{8}$ the test mass $m$ can be expressed in terms of the meter, the second and the Planck's constant $h$ as given by Eq. (1),

$$
m=K \frac{f_{J}^{2}}{g V} \times h,
$$

where $f_{J}$ is the Josephson frequency, $g$ the local acceleration of gravity, $V$ the velocity of the moving-coil, and $K$ a constant. To be able to measure a mass with a relative standard uncertainty of $10^{-8}$, it is necessary to measure each physical parameter with a better one. In this article, we present a method that will permit us to control the velocity of the moving-coil with a relative uncertainty of $10^{-9}$. In the French setup, the weight of the $500 \mathrm{~g}$ gold platinum alloy test mass will be balanced by a $5 \mathrm{~mA}$ current flowing in a circular coil of $27 \mathrm{~mm}$ diameter immersed in a $1 \mathrm{~T}$ radial magnetic field generated by a permanent magnet (SmCo). The coil and the test mass will be moved at a constant velocity of $2 \mathrm{~mm} \mathrm{~s}^{-1}$ producing a $1 \mathrm{~V}$ induced voltage at the coil terminals. The vertical displacement will be assured thanks to a set of six flexure strips monitored by a magnetic linear motor and a piezoelectric actuator.

\section{HIGH ACCURACY INTERFEROMETRIC TECHNIQUE FOR VELOCITY CONTROL}

The velocity must be measured with a traceable method with regard to the definitions of the meter and the second in SI. For this purpose, we decided to use heterodyne optical interferometry with a laser which frequency is one of those recommended by the Comité International des Poids et Mesures to define the meter. Time is measured using a high stability quartz oscillator calibrated in regard to a cesium atomic clock.

Consider a heterodyne Michelson's interferometer with a moving mirror mounted on a translation stage. Even with the better high precision translation stages, it is impossible to obtain a stability of the velocity below to $10^{-6}$ in relative value over centimetric displacement. This can be achieved only with a two-levels translation stage. The first level (TS1) has a long travel range but a low positioning accuracy rather than the second level (TS2) has a short travel range but a high sensitivity (generally a piezoelectric actuator). The aim of TS2 is to compensate the translation defects of TS1. The principle of our method is depicted in Fig. 1. A heterodyne laser source emits two orthogonally polarized beams separated in frequency by $\delta \nu=\left|\nu_{2}-\nu_{1}\right|$. The optical beams pass through the Michelson's interferometer. They are separated thanks to a polarization beamsplitter. One of the beams is retroreflected using a fixed corner cube and the other using a movable corner cube supported by a two-levels translation stage. The two beams are recombined at the output of the interferometer and mixed thanks to a polarizer resulting in a signal $s_{3}$. This signal contains the information about the velocity.

TS1 is a commercial high accuracy translation stage with its servo control system. This servo control system is independent and different compared to the control system of TS2. The velocity control of TS2 is based on the use of a high frequency phase-shifting electronic circuit. This circuit gen- 


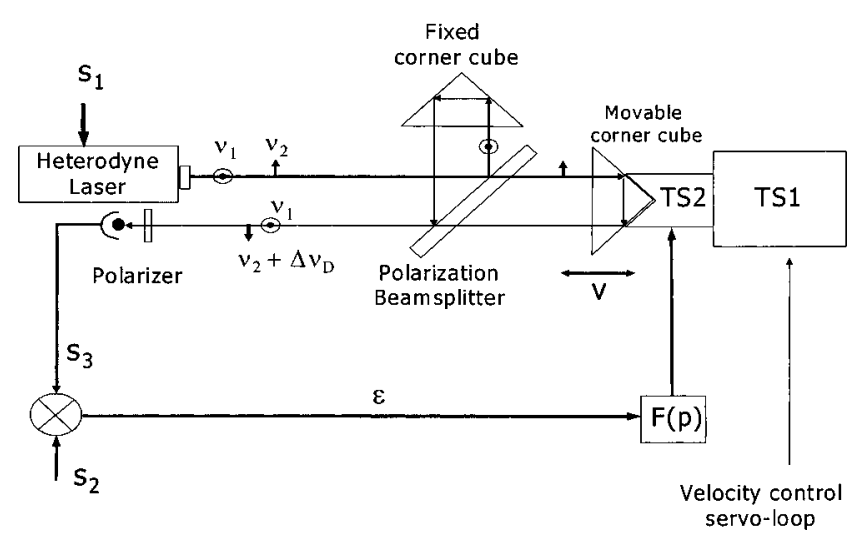

FIG. 1. Principle of the high accuracy velocity control method dedicated to the BNM watt balance experiment. A moving mirror of a Michelson's interferometer is mounted on a two-levels translation stage, one rough (TS1) and one fine (TS2). A motion of TS1 leads to a Doppler phase shift of the signal $s_{3}$ equal to $\phi_{D} \pm \delta \phi_{1}$, where $\delta \phi_{1}$ is the phase noise due to the fluctuation of $V$. Simultaneously phase shifts equal to $\phi_{D} \pm \delta \phi_{2}$ are made onto $s_{2}$ leading also to a velocity $V$ for the movable mirror but with TS2 and with an uncertainty $\delta \phi_{2} \ll \delta \phi_{1}$. The result is that the stability of the velocity of the mirror is as fine as permitted by TS2 over the entire travel range of TS1.

erates two synchronized signals $s_{1}$ and $s_{2}$ both at a frequency $\delta \nu$. It allows us to also produce phase shifts on these signals. Signal $s_{2}$ is sent to a mixer to be phase-compared with $s_{3}$. Signal $s_{1}$ feeds a Bragg cell (not seen in Fig. 1) placed in the laser head to perform the two optical beams separated in frequency by $\delta \nu$ and orthogonally polarized thanks to a birefringent plate. A motion of the mirror with a velocity $V \pm \delta V_{1}$ is generated via TS1. The motion of TS1 leads to a Doppler phase shift per unit of time of the signal $s_{3}$, equal to $\phi_{D} \pm \delta \phi_{1}$, where $\delta \phi_{1}$ is the phase noise due to $\delta V_{1}$. Simultaneously, the phase shift equal to $\phi_{D} \pm \delta \phi_{2}$ is made onto $s_{2}$ leading also to a velocity $V \pm \delta V_{2}$ for the movable mirror but with an uncertainty $\delta V_{2} \ll \delta V_{1}$. As $\delta \phi_{2} \ll \delta \phi_{1}$, signal error $\varepsilon$ is given by

$$
\varepsilon= \pm K\left(\delta \phi_{1}-\delta \phi_{2}\right) \approx \pm K \times \delta \phi_{1},
$$

where $K$ is a constant factor. Hence, the servo-loop control of TS2 will improve the defect $\delta \phi_{1}$ of TS1. The result is that $\varepsilon$ is always around zero and the stability of the velocity of the mirror is as fine as permitted by TS2 over the entire travel range of TS1. Notice that it is possible to control the direction of the displacement of the mirror by making phase shifts either on the signal $s_{2}$ either on the signal $s_{1}$. The limit of this method is due to the fact that it is not possible to equalize experimentally the two velocities perfectly. Hence the maximum travel of the movable mirror is limited by the maximum travel range of the piezoelectric actuator. However, with a $3 \mu \mathrm{m}$ travel range piezoelectric actuator and our electronics, the movable mirror has been displaced over $80 \mathrm{~mm}$ without breaking the loop lock of TS2 which is enough in the case of the French watt balance.

\section{EXPERIMENTAL SETUP}

The experimental setup is shown in Fig. 2. We use a commercial interferometric system (ZMI2001, Zygo) with a double-pass interferometer. Its resolution is equal to $0.31 \mathrm{~nm}$. The difference between the two optical components $\delta \nu$ is

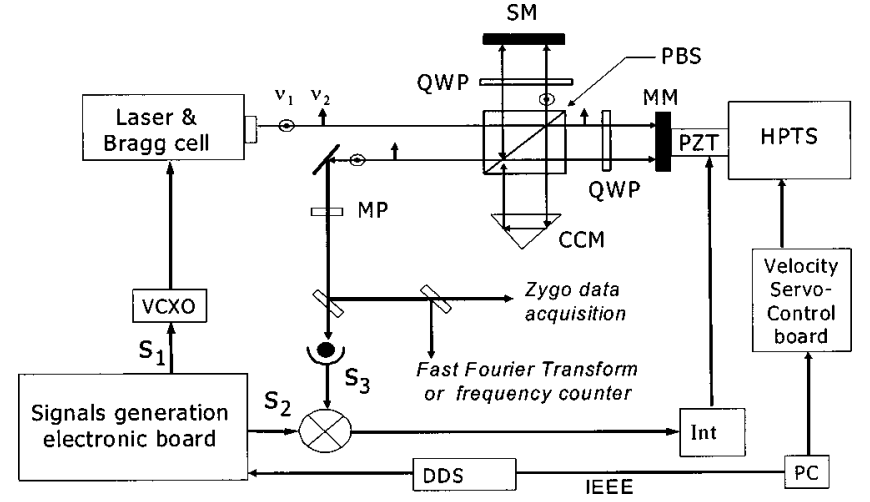

FIG. 2. Experimental setup. SM, Stationary Mirror; MM, movable mirror; PBS, Polarization Beamsplitter; QWP, Quarter Wave Plate; HPTS, High Precision Translation Stage; VCO, Voltage Control Oscillator; CCM, Corner Cube Mirror; PZT, Piezoelectric Actuator; Int, Integrator; MP, Mixing Polarizer; DDS, Digital Direct Signal generator. The part of the optical beam sent to the Fast Fourier Transform apparatus or to the frequency counter is first demodulated with a reference signal at a frequency of $20 \mathrm{MHz}$. The signal generator is controlled by a computer (PC) via an IEEE communication port.

equal to $20 \mathrm{MHz}$. The translation stage is a two-levels one. The first level TS1 is a high precision translation stage from Aerotech (ALS20010) monitored by a magnetic linear motor. It is designed to have a velocity control at the $10^{-5}$ accuracy level in relative value. The drive system is composed of a linear brushless servo motor. The total travel is about 100 $\mathrm{mm}$ and the maximum velocity is $2 \mathrm{~m} \mathrm{~s}^{-1}$. The second level $\mathrm{TS} 2$ is a piezoelectric translator (PZT). The maximum travel range is $3 \mu \mathrm{m}$ for an applied voltage of $40 \mathrm{~V}$. The velocity control of this stage is performed thanks to the method we described above. The signal generation board is made from high speed and low phase noise logic components and a high frequency clock $(640 \mathrm{MHz})$. Minimum phase shift achievable on $s_{1}$ or $s_{2}$ is equal to $2 \pi / 32$. The beam at the output of the Michelson's interferometer is divided in three parts. One part is used to generate the signal $s_{3}$ at the mixer level. Another part is sent to the Zygo data acquisition board and then transferred to a PC using a PCI-VME connection to visualize the displacement of the movable mirror. The third part of the beam is used to measure the Doppler frequency shift using either a fast Fourier transform (FFT) apparatus either a frequency counter. For this purpose, the useful signal is first demodulated with a reference signal at a frequency $\delta \nu$ (not represented in Fig. 2).

\section{RESULTS AND DISCUSSIONS}

\section{A. Spectral analysis of the servo loop control efficiency}

We measure the Doppler frequency shift during the displacement of the mirror by sending the output of the Michelson's interferometer into a FFT apparatus (SR785, Stanford) as seen in Fig. 2. Figure 3 shows the Doppler frequency shift of the optical beams during the displacement of the mirror with a velocity of $0.2 \mathrm{~mm} \mathrm{~s}^{-1}$ using only TS1. Such a velocity corresponds to a mean frequency of about 1.264 $\mathrm{kHz}$ with a dispersion due to the instability of the velocity during the displacement. Once the second level translation 


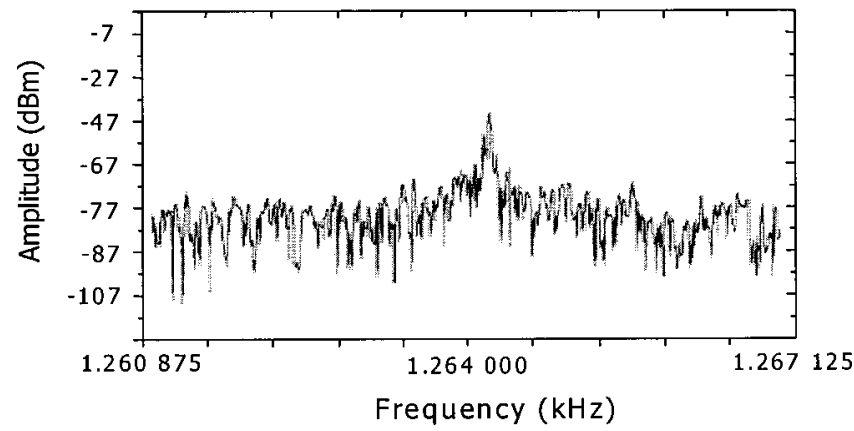

FIG. 3. Spectral measurement of the Doppler frequency shift during the displacement of the mirror. The velocity of the mirror is equal to $0.2 \mathrm{~mm} \mathrm{~s}^{-1}$. Only the rough level of the two-levels translation stage is used.

stage TS2 and its velocity control loop get started the peak becomes narrow (Fig. 4) showing hence the efficiency of our velocity control method.

\section{B. Characterization of the performance of the velocity control servo-loop using the Allan variance}

To quantify the stability of the velocity when the two control servo-loops are running, we use the Allan standard deviation. It is currently used in time frequency metrology for an estimation of the stability of primary frequency standards. ${ }^{9}$ For this purpose, the signal coming from one part of the beam at the output of the Michelson's interferometer at a frequency $\delta \nu \pm \nu_{D}$ (where $\delta \nu=20 \mathrm{MHz}$ and $\nu_{D}$ is the Doppler frequency shift) is demodulated with a reference signal at a frequency $20 \mathrm{MHz}$ coming from our high frequency electronic circuit. The frequency of the resulting signal is measured thanks to a high stability frequency counter HP53132A with a relative accuracy of $10^{-10}$. The mirror is moved over $80 \mathrm{~mm}$ with a velocity of $2 \mathrm{~mm} \mathrm{~s}^{-1}$ leading, respectively, to an acquisition time of $40 \mathrm{~s}$. During this time, the Doppler frequency shift is measured over $10 \mathrm{~s}$ giving one measurement point. During the $40 \mathrm{~s}, 3$ points are recorded. This step is repeated 34 times leading to 102 points of $10 \mathrm{~s}$. We use the overlapped Allan variance $\sigma_{v}^{2}(\tau)$ calculus. The maximum estimation time is then about $400 \mathrm{~s}$. In the same way, the overlapped Allan standard deviation for a velocity of $0.2 \mathrm{~mm} \mathrm{~s}^{-1}$ has been calculated with a set of 200 data. Each data is measured over $30 \mathrm{~s}$. The maximum estimation

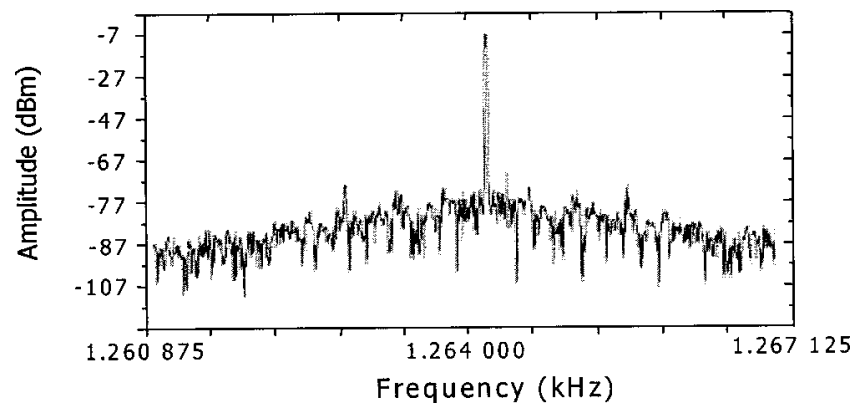

FIG. 4. Spectral measurement of the Doppler frequency shift using the two levels of control. The velocity of the mirror is equal to $0.2 \mathrm{~mm} \mathrm{~s}^{-1}$. The two levels of the translation stage are used. Once the second level translation stage and its velocity control loop get started the peak becomes narrow (by comparison to Fig. 3).

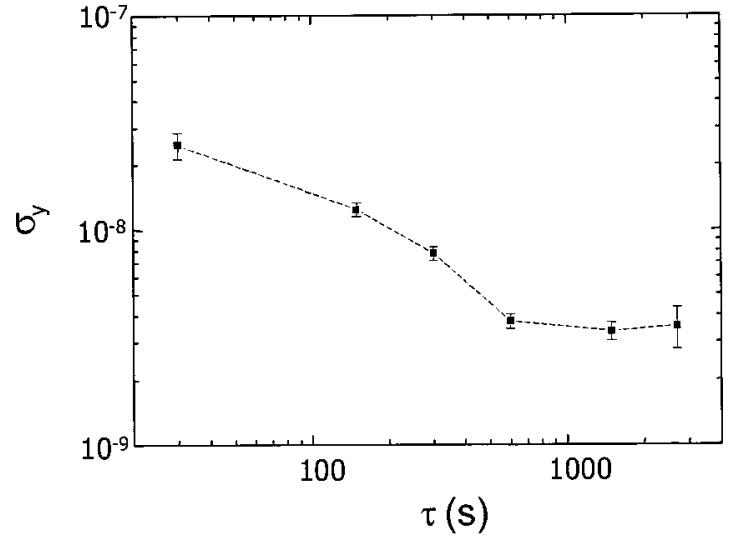

FIG. 5. Allan standard deviation of the Doppler frequency shift for a velocity of $0.2 \mathbf{~ m m ~ s}^{-1} . \sigma_{y}(\tau)$ has been calculated with 200 data. Each point is averaged over $30 \mathrm{~s}$.

time with the overlapped Allan standard deviation is then 2700 s. Figures 5 and 6 represent the standard deviation of the Doppler frequency shift corresponding, respectively, to a velocity of the moving mirror of $0.2 \mathrm{~mm} \mathrm{~s}^{-1}$ (i.e., $1.264 \mathrm{kHz}$ ) and $2 \mathrm{~mm} \mathrm{~s}^{-1}$ (i.e., $12.641 \mathrm{kHz}$ ). One can show that $\sigma_{y}(\tau)$ is about $3.5 \times 10^{-9}$ over $2700 \mathrm{~s}$ for a velocity of $0.2 \mathrm{~mm} \mathrm{~s}^{-1}$ (Fig. 5) and $2.2 \times 10^{-9}$ over $400 \mathrm{~s}$ for a velocity of $2 \mathrm{~mm} \mathrm{~s}^{-1}$ (Fig. 6). This result shows that in terms of stability, our method reaches the specifications imposed by the movingcoil watt balance project.

\section{Absolute value of the velocity}

\section{Measurement of the velocity from the Doppler frequency shift}

As we use a double-pass interferometer, the Doppler frequency shift for a velocity $V$ is equal to

$$
\nu_{D}=\nu_{0} \times \frac{4 V}{n c},
$$

where $n$ is the refractive index of air. In our case, the laser frequency has been calibrated with regard to a national reference of the Institut National de Métrologie (Paris, France) and is equal to $\nu_{0}=473.6121176 \times 10^{12} \mathrm{~Hz}$. With $V$

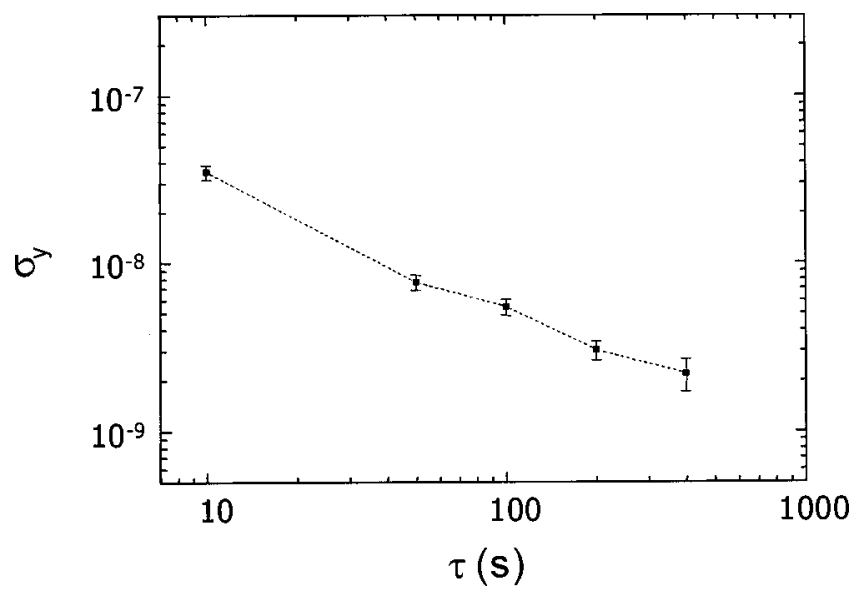

FIG. 6. Allan standard deviation of the Doppler frequency shift for a velocity of $2 \mathbf{m m ~ s}^{-1} \cdot \sigma_{y}(\tau)$ has been calculated with 100 data. Each point is averaged over $10 \mathrm{~s}$. 
$=2 \mathrm{~mm} \mathrm{~s}^{-1}$, one obtains a theoretical value (taking $n=1$ ) of the Doppler frequency shift of $12638.39980 \mathrm{~Hz}$. To compare with experimentation, we measure the Doppler frequency shift of the optical beam at the output of the Michelson's interferometer. As previously, one part of the beam stemmed from the interferometer is sent onto a detector and mixed with a reference signal at a frequency of $20 \mathrm{MHz}$. The resulting signal is sent to the frequency counter HP 53132A and the value is recorded on a computer. For this purpose, the mirror moves over $80 \mathrm{~mm}$ and the Doppler frequency shift is recorded over $40 \mathrm{~s}$. This step is repeated until we obtain 100 points. We measure an arithmetical mean value of $12641.87376 \mathrm{~Hz}$ corresponding to a velocity of $2.000549747 \mathrm{~mm} \mathrm{~s}^{-1}$. The discrepancy is first due to the refractive index of air. Actually, this experiment is not made in an air-conditioned room and the optical path difference between both arms of the Michelson's interferometer is about $1 \mathrm{~m}$. Nevertheless, as the watt balance experiment is planned to be made under vacuum this error is not significant. Second, it is impossible to impose exactly a velocity of $2 \mathrm{~mm} \mathrm{~s}^{-1}$ on TS1 because of its limited accuracy. Furthermore, what is interesting for us is not to have exactly a velocity of $2 \mathrm{~mm} \mathrm{~s}^{-1}$ but a relative uncertainty of $10^{-9}$ on $V$. The relative uncertainty on $V$ by this method could reach $10^{-10}$ if the experiment is made under vacuum.

\section{Measurement of the velocity using the number of steps}

It is also possible to know the value of $V$ using the repetition rate of the phase shifts and the value of the corresponding displacement $\Delta x$. Actually, when a phase shift $\Delta \phi$ is generated on $s_{2}$ the movable mirror will move until both phases of $s_{2}$ and $s_{3}$ become equal thanks to the lock-in electronic system as seen in Fig. 2. If these phase shifts are quantified, it becomes possible to move the mirror step by step. ${ }^{10}$ The displacement value $\Delta x$ of the mirror corresponding to the phase shift $\Delta \phi$ is

$$
\Delta x=\frac{\Delta \phi}{2 \pi} \frac{\lambda_{0}}{4 n},
$$

where $\lambda_{0}$ is the laser wavelength in vacuum and $n$ the refractive index of air. The period of repetition of the phase shifts can be controlled thanks to a high-stability quartz oscillator (DS345 - Stanford), hence we can impose an uniform speed to the moving mirror given by

$$
V=\nu^{\prime} \times \Delta x,
$$

where $\nu^{\prime}$ is the repetition rate of the phase shifts. With $V$ $=2 \mathrm{~mm} \mathrm{~s}^{-1}$ and $\Delta \phi=2 \pi / 32$ corresponding to a step value of $\Delta x=4.945246313 \mathrm{~nm}$, we obtain a theoretical value $(n=1)$ of $\nu^{\prime}=404428 \mathrm{~Hz}$. The experimental value of $\nu^{\prime}$ is determined so as to minimize the error signal $\varepsilon$ at the mixer output (see Fig. 2). We found a value of $403209 \mathrm{~Hz}$ corresponding to a velocity of $1.993971733 \mathrm{~nm}$. This experimental value is different from the value found with the previous method because these two experiments have been made at different times. The tolerance on $\nu^{\prime}$ depends on the travel range of the piezoelectric actuator and the total displacement range of the movable mirror. With our $3 \mu \mathrm{m}$ travel range PZT and for a total displacement of $80 \mathrm{~mm}$, a tolerance of $1 \mathrm{~Hz}$ on the value of $\nu^{\prime}$ is enough. This could be easily achievable with the digital signal generator DS345 from Stanford. The discrepancy between the theoretical and experimental values is essentially due to the refractive index of air and to the limited accuracy of the velocity control servo-loop TS1. One can show that the relative uncertainty on $V$ is given

$$
\frac{\sigma_{\langle V\rangle}}{\langle V\rangle}=\frac{2 \sqrt{3}\langle\Delta x\rangle^{1 / 2} \sigma_{\langle\Delta x\rangle}}{D^{3 / 2}},
$$

where $\langle\Delta x\rangle$ is the mean step value, $\sigma_{\langle\Delta x\rangle}$ the noise level on each step, and $D$ the total displacement value. Neglecting the refractive index of air, the relative uncertainty on $\langle V\rangle$ could reach $10^{-12}$ (using $\langle\Delta x\rangle=4.945297313 \mathrm{~nm}, \sigma_{\langle\Delta x\rangle}=0.22 \mathrm{~nm}$ and $D=80 \mathrm{~mm}$ ).

\section{ACKNOWLEDGMENT}

This project is funded by the Bureau National de Métrologie of France and overseen by G. Genevès of the BNM-LAMA/LNE Laboratory.

${ }^{1}$ T. Quinn, IEEE Trans. Instrum. Meas. 40, 81 (1991).

${ }^{2}$ Y. Fujii, Y. Miki, F. Shiota, and T. Morokuma, IEEE Trans. Instrum. Meas. 50, 580 (2001).

${ }^{3}$ B. P. Kibble, I. A. Robinson, and J. H. Bellis, Metrologia 27, 173 (1990).

${ }^{4}$ B. P. Kibble, Atomic Masses and Fundamental Constants, edited by J. H. Sanders and A. H. Wapstra (Plenum, New York, 1976), Vol. 5, p. 545

${ }^{5}$ V. Bego, J. Butorac, and D. Illié, IEEE Trans. Instrum. Meas. 48, 212 (1999).

${ }^{6}$ I. A. Robinson and B. P. Kibble, IEEE Trans. Instrum. Meas. 46, 596 (1997).

${ }^{7}$ C. A. Hamilton and Y. H. Tang, Metrologia 36, 53 (1999).

${ }^{8}$ B. Jeckelmann and B. Jeanneret, Rep. Prog. Phys. 64, 1603 (2001).

${ }^{9}$ D. W. Allan, H. Hellwig, and D. J. Glaze, Metrologia 11, 133 (1975).

${ }^{10}$ S. Topcu, L. Chassagne, D. Haddad, Y. Alayli, and P. Juncar, Rev. Sci. Instrum. 74, 4876 (2003). 